\section{Experiencias de mujeres sobrevivientes a morbilidad materna extrema en México, un estudio cualitativo desde el modelo de las tres demoras}

\author{
Experiences of survivors of maternal near miss \\ in Mexico: a qualitative study based on the \\ three delays model
}

\section{Experiências de mulheres sobreviventes à morbidade materna extrema em México: um estudo qualitativo partindo do modelo das três demoras}

\section{Resumen}

El objetivo fue analizar las experiencias de sobrevivientes de morbilidad materna extrema, para desde el enfoque de las tres demoras, identificar los determinantes que obstaculizan este proceso en el estado de San Luis Potosí, México. Durante 2016-2017, se realizó un estudio cualitativo con 27 mujeres que vivieron un evento de este tipo entre 2014 y 2016 en hospitales públicos. Las informantes fueron seleccionadas mediante muestreo teórico y entrevistadas en sus domicilios. El análisis de datos se realizó desde la propuesta de teoría fundamentada de Strauss er Corbin, las categorías asociadas con la primera demora fueron: (1) oportunidades pérdidas para la identificación de morbilidad materna extrema, (2) temor de comunicar el malestar frente a la descalificación, (3) entre el miedo y la fe en que algo superior resolverá, (4) desarrollo de la percepción del riesgo obstétrico. Con la segunda demora: (1) falta de ordenamiento urbano para el traslado, (2) ausencia de regulación de costos del traslado, (3) falta de claridad sobre los itinerarios y rutas críticas para acceder a la atención, (4) coordinación institucional para el traslado oportuno; y finalmente con la tercera: (1) rechazo institucional, (2) deficiencias de infraestructura, personal y equipo, y (3) entre la experiencia y la falta de ella del personal de salud. Las experiencias compartidas evidencian retos en materia de política pública y trabajo intersectorial para mejorar los resultados en materia de morbilidad materna extrema.

Near Miss Salud; Preeclampsia; Salud Materna; Urgencias Médicas; Investigación Cualitativa
Yesica Yolanda Rangel-Flores 1

Luis Eduardo Hernández-Ibarra 1

Alexia Guadalupe Martínez-Ledezma 1

Minerva García-Rangel 2

doi: $10.1590 / 0102-311 \times 00035418$

\author{
Correspondencia \\ Y. Y. Rangel-Flores \\ Facultad de Enfermería y Nutrición, Universidad Autónoma de \\ San Luis Potosí. \\ Av. Niño Artillero 130, Zona Universitaria, San Luis Potosí, CP \\ 78240, México. \\ yesica.rangel@uaslp.mx \\ 1 Facultad de Enfermería y Nutrición, Universidad Autónoma \\ de San Luis Potosí, San Luis Potosí, México. \\ 2 Unidad Académica Multidisciplinaria, Universidad Autónoma \\ de San Luis Potosí, San Luis Potosí, México.
}




\section{Introducción}

A pesar de que se han desarrollado diversas estrategias para comprender los complejos procesos implicados en la salud materna, la mayor parte de estas centra su atención en los factores contribuyentes a las muertes maternas. La "autopsia verbal", por ejemplo, identifica los factores socioculturales o institucionales que contribuyeron al deceso ${ }^{1}$, sin embargo, se han realizado menos esfuerzos para comprender los procesos que se implican en la morbilidad materna extrema, o en recuperar las experiencias vividas de mujeres que "casi han muerto" por alguna complicación asociada a la gestación 2.

La Organización Mundial de la Salud (OMS) recomienda que sean llevados a cabo más estudios con mujeres sobrevivientes de morbilidad materna extrema, puesto que trabajar con ellas posibilita acceder a un mayor número de casos para analizar, entendiendo de que por cada muerte materna se tiene un registro de cerca de 20 sobrevivientes de morbilidad materna extrema 2. En este sentido, mientras en África y Asia han comenzado a impulsarse trabajos de investigación con sobrevivientes de morbilidad materna extrema 3,4,5,6,7, en América Latina la investigación ha continuado centrándose principalmente en las muertes maternas.

Por otra parte, es pertinente mencionar que los estudios desarrollados en América Latina sobre morbilidad materna extrema continúan privilegiando la visión de profesionales, -sobre los factores que contribuyen al evento de emergencia obstétrica-, a partir de la revisión de expedientes e historias clínicas 8,9,10,11, ignorando las experiencias que las mujeres quieren contar sobre la morbilidad materna extrema.

La pertinencia del modelo de las tres demoras para el estudio de la morbilidad materna extrema radica, principalmente, en que posibilita comprender cuáles son los determinantes sociales que influyen en los procesos que viven las mujeres y sus redes sociales. Primero para identificar las señales de alarma y buscar atención médica (primera demora), después para trasladarse hasta los servicios de salud (segunda demora) y, finalmente, para acceder a una atención eficaz y eficiente (tercera demora) 12.

Las razones que inciden en las demoras para acceder a la atención institucional de las emergencias obstétricas son diversas, y tienen relación con las condiciones culturales, sociales y políticas que prevalecen en cada contexto. Un estudio realizado en Asia documentó que la segunda demora fue la más relevante, asociada con la carencia de programas de protección de salud 3 . Otro estudio llevado a cabo en Ruanda señaló también la segunda demora como la más relevante, sin embargo, las razones asociadas fueron la ausencia de apoyos en materia de protección en salud, pero sobre todo, el temor de las mujeres para acercarse a los servicios sanitarios por la criminalización del aborto 5 .

Un estudio realizado en Mozambique señaló la segunda demora como la menos relevante, destacando la predominancia de la primera demora y asociando esta con la escasa adherencia a la atención prenatal 4. En Pretoria (Sudáfrica), un estudio destacó la importancia de las demoras I y III, la primera asociada con la falta de conocimiento sobre las señales de alarma y la ausencia de calidad en el cuidado prenatal, la tercera con el retraso en la admisión y la atención deficiente 7 .

En América Latina se identificaron dos estudios relevantes, uno desarrollado en Argentina y otro en México, el primero reportó evidencias en los tres tipos de demoras, sin predominio de alguna de ellas en particular 13; el segundo enfatizó que las demoras I y III se presentaron en la mayoría de los casos 14 .

Enmarcado en lo descrito anteriormente, y en función de que el modelo de las tres demoras ha demostrado ser útil para identificar los determinantes que inciden en la búsqueda y acceso oportuno a la atención profesional de la emergencia obstétrica, -dentro de contextos particulares-, el objetivo de este estudio fue analizar las experiencias de sobrevivientes de morbilidad materna extrema, para identificar los determinantes que obstaculizan este proceso en el estado de San Luis Potosí, México, desde este enfoque. 


\section{Material y métodos}

Estudio cualitativo implementado durante 2016-2017. Se entrevistó a 27 mujeres sobrevivientes de morbilidad materna extrema, seleccionadas mediante muestreo teórico. Los criterios de selección se determinaron en el marco de los criterios de clasificación de near miss propuestos por la OMS 15. En el Cuadro 1 pueden apreciarse dichos criterios.

El número de participantes se determinó en función del criterio de saturación teórica, realizando tantas entrevistas como fueran necesarias, hasta que no se identificara la emergencia de datos nuevos, y las relaciones entre las categorías teóricas propuestas quedaran bien establecidas y validadas para asegurar la lógica interna de los datos 16 .

Las participantes fueron contactadas mediante visita domiciliaria y se obtuvieron los consentimientos informados, con el fin de cumplir con los estándares éticos y legales que establecen los documentos normativos nacionales e internacionales en investigación en salud. Se planteó realizar la suspensión de la entrevista ante una crisis emocional, situación que no se presentó. El estudio fue aprobado y monitoreado por el Comité Estatal de Ética e Investigación de los Servicios de Salud de San Luis Potosí, con registro SLP/002/2017.

La información se obtuvo mediante entrevista individual, basada en un guion semiestructurado enmarcado en las tres demoras, a medida que se avanzó en la realización de estas, el guion se volvió a trabajar, con el fin de profundizar en las categorías que fueron construyéndose durante un proceso de comparación constante 17 . En el Cuadro 2 pueden apreciarse algunas de las preguntas que se realizaron en las entrevistas.

Las entrevistadoras fueron las tres investigadoras que figuran como autoras y fueron audiograbadas, previo consentimiento informado. El análisis de los datos se realizó desde la propuesta de teoría fundamentada de Strauss \& Corbin 16. De manera manual fueron aplicadas las estrategias de codificación abierta, axial y selectiva.

Para la codificación abierta, las entrevistas fueron transcritas íntegramente en Micfrosoft Word 2016 (https://products.office.com/) y revisadas varias veces hasta reducir la información a 517 códigos. En la etapa de codificación axial, los códigos se pasaron al programa Microsoft Excel 2016 (https:// products.office.com/), con el fin de relacionar los códigos dentro de subcategorías establecidas desde la teoría y la experiencia empírica de los investigadores en el tema de morbilidad materna extrema. Finalmente, en la codificación selectiva, se agruparon las subcategorías (que contenían los códigos vivos) dentro de tres categorías seleccionadas, a partir de la propuesta de Thaddeus \& Maine 12. En el Cuadro 3 pueden consultarse la relación entre categorías y subcategorías.

\section{Cuadro 1}

Criterios para la validación de casos de morbilidad materna extrema.

\begin{tabular}{|l|c|}
\hline Indicador & Criterios de inclusión \\
\hline Diagnóstico & Preeclampsia severa, eclampsia, hemorragias posparto severas, sepsis y ruptura uterina \\
\hline Disfunción orgánica & Cardiaca, vascular, inmunológica, respiratoria, renal, hepática, metabólica hematológica y cerebral \\
\hline Intervenciones críticas & Transfusión de más de tres paquetes de hemoderivados, radiología de intervención y laparotomía (incluye \\
histerectomía, excluye cesárea)
\end{tabular}

Fuente: criterios de determinación de morbilidad materna extrema, propuestos por la Organización Mundial de la Salud 1. 


\section{Cuadro 2}

Preguntas incluidas en el guion de entrevista semiestructurada basada en el modelo de las tres demoras.

\begin{tabular}{|c|c|}
\hline Primera demora & $\begin{array}{l}\text { ¿Qué le hizo pensar que algo no andaba bien en su embarazo (signos, síntomas, presentimiento, actitud diferente en los } \\
\text { demás)? } \\
\text { ¿Cómo fue el proceso inmediato de buscar ayuda? (a quién recurrió, cómo actuó esa o esas personas, quién tomó la } \\
\text { decisión de acudir a los servicios de salud). }\end{array}$ \\
\hline Segunda demora & $\begin{array}{l}\text { ¿Cómo fue el proceso de traslado hasta los servicios de salud? (medios de transporte disponibles, tiempo real invertido } \\
\text { en el traslado, obstáculos o facilidades identificadas, persona en quien recayó tomar la decisión de dónde buscar ayuda, } \\
\text { respuesta de las redes familiares o comunitarias). } \\
\text { ¿Qué coincidencias o diferencias existieron entre el camino que siguió para buscar la atención y su plan de parto (si es } \\
\text { que lo habían elaborado en conjunto con el personal sanitario)? }\end{array}$ \\
\hline Tercera demora & $\begin{array}{l}\text { ¿Cómo fue el proceso que vivió desde que llegó al hospital y la solución de su complicación? (quiénes intervinieron como } \\
\text { facilitadores u obstaculizadores para la atención oportuna, tiempo de espera, razones de los retrasos, percepción de la } \\
\text { calidad de la atención). }\end{array}$ \\
\hline
\end{tabular}

Fuente: guión de entrevista semiestructurado, creado por los autores.

\section{Cuadro 3}

Descripción de las categorías y su asociación a subcategorías de análisis.

\begin{tabular}{|c|c|c|}
\hline Categoría & Definición teórica de la categoría & Subcategorías identificadas \\
\hline Primera demora & $\begin{array}{l}\text { Implica la demora en decidir buscar atención: la decisión de } \\
\text { buscar ayuda es el primer paso para recibir cuidados obstétricos } \\
\text { de emergencia. Depende de las capacidades y oportunidades } \\
\text { de la mujer y su entorno para reconocer una complicación que } \\
\text { amenaza la vida, así como de la información de la que dispone } \\
\text { acerca de dónde puede acudir }\end{array}$ & $\begin{array}{l}\text { 1) Oportunidades perdidas para la identificación de } \\
\text { morbilidad materna extrema } \\
\text { 2) Temor de comunicar el malestar frente a la } \\
\text { descalificación } \\
\text { 3) Entre el miedo y la fe en que algo superior resolverá } \\
\text { 4) Desarrollo de la percepción del riesgo obstétrico }\end{array}$ \\
\hline Segunda demora & $\begin{array}{l}\text { Implica la demora en identificar y acceder a un servicio de salud: } \\
\text { depende de la distancia al servicio de salud, la disponibilidad y } \\
\text { eficiencia del transporte y el costo de los traslados }\end{array}$ & $\begin{array}{l}\text { 1) Falta de ordenamiento urbano para el traslado } \\
\text { 2) Ausencia de regulación de costos del traslado } \\
\text { 3) Falta de claridad sobre los itinerarios y rutas críticas } \\
\text { para acceder a la atención } \\
\text { 4) Coordinación institucional para el traslado oportuno }\end{array}$ \\
\hline Tercera demora & $\begin{array}{l}\text { Implica la demora en obtener el tratamiento adecuado y } \\
\text { oportuno. La provisión de cuidados obstétricos de emergencia } \\
\text { depende del número y la disponibilidad de personal capacitado, } \\
\text { de medicamentos, suministros e insumos (antibióticos, sulfato } \\
\text { de magnesio, sangre segura) y de la infraestructura (quirófanos) } \\
\text { del servicio de salud }\end{array}$ & $\begin{array}{l}\text { 1) Rechazo institucional } \\
\text { 2) Entre la experiencia y la falta de ella del personal de } \\
\text { salud } \\
\text { 3) El trato digno en la reconstrucción de su experiencia }\end{array}$ \\
\hline
\end{tabular}




\section{Resultados y discusión}

\section{Caracterización de la población}

El rango de edad fue de 16 a 41 años, con un promedio de 26. La mayoría (85,6\%) vivía con su pareja, cuando se produjo la experiencia de morbilidad materna extrema, 53,5\% en unión libre y 32,1\% en matrimonio. La mayoría refirió tener la primaria completa $(32,1 \%), 17,8 \%$ la primaria incompleta y el nivel más alto reportado fue la licenciatura incompleta que representó el 10,7\% de los casos. Las ocupaciones más reportadas fueron ama de casa con 35,7\% y empleada con $21,4 \%$.

La totalidad de las informantes fueron atendidas en hospitales públicos y contaban con Seguro Popular, sistema de protección a población no derechohabiente, público y voluntario. El 55,5\% de las informantes eran primigestas en el momento de la experiencia de morbilidad materna extrema, casi la mitad de ellas (44,4\%) estaban entre la 38 y 40 semanas de gestación, en lo que se refiere al seguimiento de médico prenatal, 77,7\% de ellas había recibido entre 5 y 8 consultas prenatales, 18,5\% lo inició hasta el segundo trimestre y $3,7 \%$ hasta el tercero. Variables como el ser primigestas y la tardía incorporación a monitoreo prenatal han sido referidas como condicionantes que prevalecen en población que experimenta morbilidad materna extrema 18,19 .

Algunos antecedentes para contextualizar el evento de morbilidad materna extrema son recuperados en la Tabla 1, donde puede apreciarse que los trastornos hipertensivos predominaron, lo que coincide con lo encontrado en la mayoría de los estudios sobre el tema 20,21.

\section{Primera demora}

La totalidad de las informantes refirió que en su experiencia de morbilidad materna extrema eran beneficiarias del Seguro Popular, un sistema de protección social en salud que se ha implementado en México para garantizar que la población, que no cuenta con seguridad social, acceda a servicios de salud. Lo anterior resulta en un contraste con lo que han reportado estudios realizados en Asia y África, en los que la pobreza y la ausencia de protección en salud constituyen el principal obstáculo

\section{Tabla 1}

Caracterización de la morbilidad materna extrema en las informantes.

\begin{tabular}{lcc}
\hline Criterio & $\mathbf{n}$ & $\%$ \\
\hline Diagnóstico médico & & \\
$\quad$ Preeclampsia severa & 15 & 55,5 \\
Eclampsia & 3 & 11,1 \\
Sepsis & 2 & 7,4 \\
Ruptura uterina & 1 & 3,7 \\
Hemorragia posparto severa & 6 & 22,2 \\
Disfunción orgánica & & 3,7 \\
Hepática & 1 & 7,4 \\
Renal & 2 & 7,4 \\
Cardiaca & 2 & 11,1 \\
Cerebral & 3 & 7,4 \\
Respiratoria & 2 & \\
Manejo médico & & 92,5 \\
Ingreso a Unidad de Cuidados Intensivos & 25 & 30,0 \\
Transfusión & 8 & 15,0 \\
Histerectomía & 4 & 3,7 \\
Laparatomía & 1 &
\end{tabular}

Fuente: datos obtenidos de los expedientes y diarios de código mater, confirmados con las mujeres a través de entrevista. 
para acceder a la salud materna, no sólo en lo que se refiere al acceso a hospitales de segundo y tercer nivel, sino incluso para recibir atención prenatal 3,4.

Las narrativas permitieron confirmar la hipótesis, que han planteado otros investigadores, respecto a que la presencia de una mujer embarazada en los hogares determina de manera importante la búsqueda de la afiliación 22. Con respecto a lo planteado anteriormente, se identificó que varias de las participantes buscaron afiliarse, hasta que se supieron embarazadas, ello implicó un control prenatal tardío en un importante número de casos $(22,2 \%)$.

\section{- Oportunidades perdidas para la identificación de morbilidad materna extrema}

Las narrativas de las informantes posibilitaron darse cuenta, por otra parte, que el garantizar el acceso a la salud prenatal no siempre es suficiente. Algunas refirieron como, pese a haber buscado atención médica ante la intuición de que algo no iba bien en sus embarazos, el personal médico devaluó la relevancia de sus manifestaciones, o bien, las contempló como condiciones fisiológicas propias del embarazo. Esto puede apreciarse en la siguiente narrativa:

"Me empecé a dar cuenta porque subí mucho de peso y me sentía mal, yo sabía que no era normal, no comía de más, pero despuesito de los 5 meses subí como 4 kilos y ya cuando fui a la última consulta pesaba ya 70 kilos, el doctor me regañó y me dijo que me iba a mandar con la nutrióloga, porque yo había subido mucho para un mes (...). Yo le decía que me veía hinchada y él me decía que era normal en el embarazo, que era que no caminaba y que me pusiera a caminar" (P. 3, 17 años).

Incluso se identificó la existencia de limitaciones por parte del personal médico para identificar el riesgo y las manifestaciones de morbilidad materna extrema en mujeres con antecedentes de morbilidad materna extrema en embarazos previos.

\section{- Temor de comunicar el malestar frente a la descalificación}

El miedo a ser juzgadas o ignoradas durante las revisiones médicas fue una constante que parece haber afectado su confianza para comunicar sus malestares. Pero la tendencia a descalificar sus intuiciones o sintomatología no se acotó exclusivamente al personal médico, sus redes sociales y familiares también deslegitimaron o subestimaron las sensaciones de las mujeres, respecto a que algo no estaba bien en sus embarazos.

"Me decían en el centro de salud que era normal en el embarazo que uno se hinchara, también dijo mi mamá que eso era normal, ella y mis hermanas también se habían hinchado, me decían lo que pasa es que te quieres consentir, eres una exagerada"'(P. 7, 23 años).

La tendencia a normalizar las molestias o el sufrimiento durante el embarazo encuentra sentido en un contexto como el latinoamericano, en el que históricamente se ha contemplado el embarazo, el parto y hasta la maternidad, como procesos que las mujeres "deben sufrir", puesto que para ello han sido creadas (por lo divino) y criadas (desde lo social).

Algunas narraron no haber sido apoyadas por sus redes para el traslado hacia los servicios médicos, sino hasta que presentaron complicaciones mayores, convulsiones, vómitos o cuando perdieron la capacidad para caminar por sí mismas.

\section{- Entre el miedo y la fe en que algo superior resolverá}

Fueron diversas las razones que las mujeres enunciaron para postergar el traslado, la más mencionada fue el miedo. Se identificó que algunas mujeres que sustentan su falta de acción para acercarse a los servicios de salud en la fe de que algo superior e infalible, les protegerá y la situación se resolverá sin la intervención médica.

"No me daba miedo dejar a mis hijos solos, soy creyente le digo, y si Dios me permitía tener otro bebé, siento que, o sea que no es por nada y que no me va a dejar a mis hijos solos, o sea, que si me permite tener a otro bebe es porque puedo criarlo y por qué voy a poder estar con él" (P. 1, 33 años). 


\section{- Desarrollo de la percepción del riesgo obstétrico}

Las oportunidades a las que acceden las mujeres para construir su percepción en torno al riesgo obstétrico es determinante en la identificación oportuna de las señales de alarma, y en la toma de decisión de acercarse a los servicios de salud. La comunicación que el personal médico establece con las mujeres, dota a estas de seguridad sobre hacia dónde trasladarse, y aumenta la confianza que estas sienten hacia los servicios.

"...Si me sentía mal me iba sola, el doctor pues, me daba hojas de los signos de dolor, de contracciones, de los síntomas, cuando yo los sentía yo me iba, porque él [médico] ya me habia explicado cuando irme y a dónde irme" (P. 9, 18 años).

La manera en que el personal habla sobre los riesgos asociados a morbilidad materna extrema puede tener resultados ambivalentes, es decir, tanto puede desarrollar su agencia para buscar con urgencia la atención profesional, como pueden generar un miedo que las incapacita para tomar decisiones en el momento de la emergencia. Se identificó que las mujeres que refirieron haber recibido información suficiente y clara, y no sólo los resultados negativos de las complicaciones, sino también sobre los tratamientos disponibles para garantizar la atención, narraron menos ansiedad y miedo en el momento de tomar la decisión de buscar ayuda profesional. Por el contrario, las mujeres cuya comunicación del riesgo se centró sólo en sentenciarlas sobre que "debían cuidarse para no morir ellas o sus hijos", narraron emociones (miedo, culpa, temor) que retrasaron la búsqueda de ayuda, algunas preferían esperar para el traslado, con la expectativa y la fe de que "la molestia" desaparecería sin intervención médica.

Como ha podido observarse, en esta primera demora se identificó que el personal sanitario del primer nivel de atención presenta debilidades en la competencia clínica para detectar los signos cardinales asociados a morbilidad materna extrema, incluso en mujeres que han sufrido esta condición en embarazos previos; esta situación ha sido reportada en otros estudios realizados en torno al tema 3,23 . Otro de los obstáculos, identificados en esta primera demora, tiene relación con la tendencia del personal sanitario a subestimar la sintomatología o la intuición de las mujeres sobre que algo no va bien con sus embarazos, lo cual conviene problematizar, en función de la existencia de un sistema sanitario cada vez más tecnificado, en el que como refieren algunos autores se habla mucho menos con los sujetos, ignorando lo que ellos tienen que decir respecto a sus malestares 24 .

El soporte social que narraron respecto a sus redes más cercanas, hace evidente que es necesario fortalecerlas, toda vez que prevalece no sólo una ausencia de conocimiento de las señales de alarma, sino también una falta de sensibilización para brindar un apoyo emocional y práctico real, lo cual resulta relevante, dado que es contrario a lo que otros estudios han referido al respecto, y que aseveran la existencia de redes de apoyo altamente funcionales 25 .

En función de esta ausencia de redes funcionales, resulta entendible que las mujeres prefieran refugiarse en Dios antes que en sus familiares, lo que ha sido reconocido también por otros autores 26. En este estudio pudo reiterarse que la fe y las creencias asociadas a lo divino juegan un papel importante en la respuesta que las mujeres tienen frente a la emergencia obstétrica, sin embargo, contrario al impacto positivo que otros autores han adjudicado a la fe 27 , en este estudio, refugiarse en la fe contribuyó al retraso en la llegada a los centros hospitalarios.

\section{Segunda demora}

\section{- Falta de ordenamiento urbano para el traslado}

Los obstáculos geográficos han sido asociados con la ruralidad, ya que se ha documentado que las ciudades cuentan con una cartera más amplia de instituciones de salud 28 , en este sentido, los resultados de este estudio invitan a problematizar como en las ciudades altamente urbanizadas, la isócrona calculada (minutos que lleva el trasladarse en función de la cantidad de kilómetros) se ve afectada por la ausencia de ordenamiento territorial.

Varias mujeres narraron sentirse afortunadas de que su situación de emergencia no se produjera un día de mercado o en el horario en que las principales vías están congestionadas, de ser así, piensan 
que no habrían sobrevivido. Por su parte, otras mujeres relataron haber tenido que sortear atascos de tráfico, en condiciones climáticas adversas, que se agravaban al suceder en áreas con drenajes y pavimentación deficiente, lo que hacía intransitables las vías más cortas.

"De la casa al hospital fueron como 40 minutos, mi esposo se salió a agarrar el taxi, le habló a uno y tardó mucho en llegar, ese día estuvo lloviendo, había mucho tráfico, porque estas calles de aquí se llenan de agua, había muchos carros y muchos tráiler" (P. 2, 23 años).

\section{- Ausencia de regulación de los costos del traslado}

Una de las informantes, que habitaba en una comunidad medianamente lejana del único hospital de la región, pudo llegar en una ambulancia que costeó gracias al dinero que su madre consiguió entre vecinos. Lo anterior se complicó dentro de un contexto en los que parece no haber regulación sobre los costos que estos servicios pueden generar en la población. La joven había comenzado con convulsiones, y la ambulancia no estaba dispuesta a hacer el traslado, si no se les garantizaba el pago.

"Mi mamá fue con una señora, le fue a decir que si podía llamar a una ambulancia, la ambulancia vino pero cobraba 200 pesos por subirme al centro de salud, entonces me llevó ahí [centro de salud] y ahí me dijeron que no me podía quedar ahí, la ambulancia le dijo a mi mamá que eran 400 y que si no traía el dinero nos iban a dejar hasta ahí, mi mamá los consiguió (...) Al final nos cobraron 600 [pesos]” (P. 25, 16 años).

\section{- Falta de claridad sobre los itinerarios y rutas críticas para acceder a la atención}

Muchas mujeres narraron itinerarios largos, complicados e inecesarios, que tuvieron razón en la ausencia de claridad que tenían sobre la oferta de servicios en el estado, así como con la ausencia de un plan de parto, aunque por su número de semanas de gestación deberían haberlo desarrollado junto a su médico.

Varias de las mujeres acudieron a hospitales que no contaban con condiciones necesarias para intervenir de manera segura casos de morbilidad materna extrema, perdiendo horas valiosas para garantizar la supervivencia y de los cuales salieron solas, sin acompañamiento, y sin referencia institucional hacia la búsqueda de otro servicio, con los riesgos que esto implicaba.

"Yo no sabía a cuál irme, entonces dije 'Me voy para el hospital X' me habian dicho que allí había menos gente, pero no me pasaban y yo andaba bien hinchada y me habian empezado los dolores, me fui como a las 1 , pero de ahí me salí como a las 5 al hospital del Niño y la Mujer, llegué como a las seis de la tarde y me atendieron a las 7”' (P. 11, 18 años).

\section{- Coordinación institucional para el traslado oportuno}

Algunas informantes reconocieron haberse visto beneficiadas de un verdadero compromiso para acercarlas a la atención especializada. Tal es el caso de una mujer que narró haber sido informada de que debía trasladarse al hospital, cuestión que consideró hacer después de regresar a casa, alistar pertenencias y esperar a su pareja para que le acompañara a hospitalizarse. Lo que la mujer no esperaba es que el personal del centro de salud se comunicara con el hospital, y que al darse cuenta que aún no se ingresaba, enviarían una médica para cerciorarse que la paciente saliera rumbo al hospital.

"Mandaron otra doctora de la jurisdicción, que viniera a ver por qué todavía no me iba [al hospital], o sea, que porque yo tenía que estar ya allá en el hospital, me habian dicho que tenía que ir directamente al hospital, le digo que me tenían bien vigilada" (P. 16, 31 años).

En esta segunda demora ha podido identificarse que la falta de ordenamiento territorial constituye una amenaza para el traslado, dentro de un contexto en el que la ausencia de acciones del estado para determinar el uso del territorio y la caracterización de este como producto social, no sólo determinan los procesos de salud y enfermedad 29 , sino que emerge como una variable que se contrapone a los traslados de emergencias médicas.

La falta de ordenamiento territorial y la ausencia de regulación de los costos de servicios de traslado contribuyen, -como ya han afirmado también otros autores-, a que los peores pronósticos en los eventos de emergencia obstétrica se asocien con mayores índices de marginación y pobreza 30,31. Esta relación tiene que ver con el hecho de que las mujeres en condiciones de pobreza tienen menos recur- 
sos para el traslado, -y agregaríamos-, a que su bajo nivel de alfabetización las hace más vulnerables frente al abuso en los costos del transporte disponible.

Finalmente, la preocupación y actuación del personal de atención primaria por asegurar la llegada de las mujeres a hospitales, según el nivel de atención, ha sido referida en otros estudios, evidenciando la participación de un personal sanitario que procura no sólo la atención fisiológica, sino que motiva a las mujeres para involucrarse en la garantía de su propia seguridad frente el evento 13,32.

\section{Tercera demora}

\section{- Rechazo institucional}

Varias mujeres dijeron haber sido enviadas de vuelta a sus casas porque no mostraban un cuadro lo suficientemente claro de morbilidad materna extrema. Una de las mujeres fue retornada a su domicilio con cifras elevadas de presión arterial, siendo su capacidad de agencia para comprender la enfermedad, lo que posibilitó que volviera al hospital antes de una complicación mortal.

"Me checó [la enfermera] y la traía alta, y le digo isale alta verdad? y me dice 'Sí, sale alta' y le digo 'Es necesario que me consulte? [Pasar a revisión con el médico]' Y dice 'si tú quieres le puedo hablar al doctor', entonces le digo 'ipero si es necesario?' y me dice 'no, no es necesario' entonces yo me regreso pero para en la noche cuando regresamos [al hospital] ya está otra enfermera, otro doctor y otro enfermero, ellos me dicen que por qué no había ido desde temprano y les explico que si había ido pero me regresaron, entonces el doctor se muestra molesto" (P. 14, 22 años).

\section{- Deficiencias de infraestructura, la disposición de quirófanos para someterse a una cesárea o la ausencia de incubadoras}

Las principales limitaciones institucionales identificadas por las mujeres se relacionaron con la deficiencia de infraestructura, la disposición de quirófanos para someterse a una cesérea o la ausencia de incubadoras; y contrario a otros estudios, no fueron narradas insuficiencia de medicamentos o sangre disponible 6. Algunas de las mujeres percibieron desde su ingreso a la institución, insuficiencia de espacios y sobredemanda para la atención, y asociaron con ello, el retraso de la respuesta médica ante su particular situación. En el sentido anterior, y contrario a lo que han reportado otros estudios, las mujeres no se narraron confiadas en las capacidades institucionales para resolver sus casos 28 .

"Habia mucha gente, bastante gente, de hecho, no había ni camillas para atender a alguien, yo estuve mucho tiempo (...) este, donde atienden a las mujeres (...). Si dudé quedarme ahí, no me pasaban, pero, de hecho, estaba el conflicto del seguro [popular], decían que ya no estaban atendiendo para aliviarse en el central" (P. 7, 20 años).

\section{- Entre la experiencia y la falta de ella del personal de salud}

Aunque varias reconocieron haber sido referidas tardíamente desde el primer nivel de atención, este retraso en el diagnóstico parece olvidarse, en función de que fueron los mismos servicios públicos de salud quienes salvaron su vida y las de sus hijos. Algunas se evidenciaron confundidas al narrar como, si bien la existencia de personal no capacitado retrasó su atención, otro personal (de los mismo servicios públicos de salud) mostró tener el conocimiento y la experiencia necesaria para salvar su vida.

"Hasta que llegó el doctor de la mañana y se molestó que porque me habian dado otro medicamento que no era, y que yo no estaba para parto normal, me dijo 'ay señora es que usted es cesárea y le pusieron otro medicamento que no era' y ya estuvieron ahí comentado los doctores que la doctora la habia regado y que no sé qué, tuve la suerte de que llegara él..." (P. 2, 23 años).

Que las mujeres se narren satisfechas con la calidad de la atención recibida, aun cuando hayan vivido situaciones de diagnóstico fallido o retraso en el manejo, ha sido referida por otros estudios 26,27 que han señalado como, tras la tragedia, la experiencia negativa tiende a suprimirse, debido a que se prioriza el hecho de que el personal les salvó la vida. Sin embargo, confirma lo que han aseverado otros estudios de que, en general, en América Latina persiste un bajo nivel de competencia para el tamizaje eficiente de los datos de morbilidad materna extrema en los servicios de urgencias obstétricas dentro de los hospitales de segundo nivel de atención 13,32. 
Las narraciones permiten respaldar lo que otros estudios han señalado respecto a que la percepción de las mujeres, sobre los factores que constribuyen a los buenos resultados, -en este caso la sobrevivencia-, se adjudica principalmente a la existencia de infraestructura adecuada o al personal capacitado, aunque alguna de estas dos variables no esté presente en la atención 29.

\section{Limitaciones del estudio}

Se identificó una dificultad significativa en las mujeres para reconstruir la experiencia asociada a la tercera demora. Muchas de ellas tuvieron que apoyarse de sus madres o pareja para reelaborar los procesos de atención médica, que vivieron desde su llegada al servicio de urgencias, y la resolución de la complicación, dado que la mayor de ellas, durante la etapa crítica de la complicación, dejaron de recibir información sobre su propia evolución, o bien, se vieron afectadas en su estado de conciencia para recordarlo. Llamó la atención, por otra parte, el hecho de que hasta el momento de la entrevista se atrevieron a platicar en profundidad con sus familiares sobre la experiencia vivida, antes lo habían evitado, porque tanto ellas, como sus redes de apoyo, pensaron que era innecesario y doloroso retomar la experiencia.

\section{Conclusiones}

Esta investigación permitió analizar las experiencias de sobrevivientes a morbilidad materna extrema desde el enfoque de las tres demoras. El estudio reveló que en las experiencias narradas por las sobrevivientes prevalecieron determinantes asociados a la primera demora, particularmente relacionados con las limitaciones del personal sanitario del primer nivel de atención para identificar los signos cardinales de la morbilidad materna extrema, la descalificación de los malestares de las mujeres, las creencias religiosas y la fe de que algo superior las protegerá y resolverá su condición, así como las limitaciones para construir una percepción del riesgo a partir de los discursos sanitarios.

Los determinantes asociados a la segunda demora fueron los que aparecieron con menor frecuencia. Destacó la ausencia de ordenamiento territorial, como una variable que compromete el traslado eficiente en la condición de emergencia, el abuso y la falta de regulación de los medios de transporte, y la ausencia de claridad de las sobrevivientes para la ruta crítica a seguir. En lo que respecta a los determinantes asociados a la tercera demora, se identificó que existe una seria dificultad en las mujeres para reelaborar esta etapa, las experiencias más significativas en esta demora tuvieron que ver con el rechazo institucional por el inadecuado tamizaje durante el triage obstétrico. 


\section{Colaboradores}

Y. Y. Rangel-Flores participó de la elaboración de protocolo e instrumentos de recolección de información, recolección de información, análisis de resultados y elaboración del artículo. L. E. Hernández-Ibarra colaboró en la elaboración de protocolo de investigación, revisión de los instrumentos de recolección de información, análisis de los datos y elaboración del artículo. A. G. Martínez-Ledezma participó en la elaboración de protocolo, recolección de información, transcripción de las entrevistas y elaboración del artículo. M. García-Rangel colaboró en la elaboración de protocolo, recolección de información y elaboración del artículo.

\section{Informaciones adicionales}

ORCID: Yesica Yolanda Rangel-Flores (00000001-5673-6891); Luis Eduardo Hernández-Ibarra (0000-0002-3889-7533); Alexia Guadalupe Martínez-Ledezma (0000-0002-6643-5301); Minerva García-Rangel (0000-0002-9338-0617).

\section{Agradecimientos}

Gracias a las mujeres que con sus historias nos posibilitan proponer más políticas en materia de salud, pertinentes a sus realidades. A los servicios de salud del estado de San Luis Potosí, por las facilidades otorgadas para localizar a las participantes. Al del Programa para el Desarrollo Profesional Docente para el Tipo Superior (PRODEP) por los recursos para la investigación.

\section{Referencias}

1. Organización Mundial de la Salud. Más allá de las cifras, revisión de las muertes maternas y las complicaciones del embarazo para hacer la maternidad segura. Ginebra: Organización Mundial de la Salud; 2009.

2. Centro Latinoamericano de Perinatología, Salud de la Mujer y Reproductiva. Plan de acción para acelerar la reducción de la mortalidad materna y la morbilidad materna grave: estrategia de monitoreo y evaluación. Montevideo: Centro Latinoamericano de Perinatología, Salud de la Mujer y Reproductiva; 2012.

3. Mohammadi S, Carlbom A, Taheripanah R, Essén B. Experiences of inequitable care among Afghan mothers surviving near-miss morbidity in Tehran, Iran: a qualitative interview study. Int J Equity Health 2017; 16:121.

4. David E, Machungo F, Zanconato G, Cavaliere E, Fiosse S, Sululu C, et al. Maternal near miss and maternal deaths in Mozambique: a crosssectional, region-wide study of 635 consecutive cases assisted in health facilities of Maputo province. BMC Pregnancy Childbirth 2014; 14:401.

5. Påfs J, Musafili A, Binder P, Klingberg M, Rulisa S, Essén B. Beyond the numbers of maternal near-miss in Rwanda, a qualitative study on women's perspectives on access and experiences of care in early and late stage of pregnancy. BMC Pregnancy Childbirth 2016; 16:257.

6. Hussein J, Hirose A, Owolabi O, Imamura M, Kanguru L, Okonofua F. Maternal death and obstetric care audits in Nigeria: a systematic review of barriers and enabling factors in the provision of emergency care. Reprod Health 2016; 13:47.

7. Soma-Pillay P, Pattinson RC. Barriers to obstetric care among maternal near misses. S Afr Med J 2016; 106:1110-3.

8. Nava M, Urdaneta JR, González ME, Labarca L, Silva A, Contreras A, et al. Caracterización de la paciente obstétrica críticamente enferma, experiencia de la maternidad "Dr. Armando Castillo Plaza”, Maracaibo, Venezuela: 20112014. Rev Chil Obstet Ginecol 2016; 81:88-96.

9. Cardoso M, Soares T, Silveira A. Fatores determinantes do near miss materno em uma unidade de terapia intensiva obstétrica. Rev Bras Ginecol Obstet 2016; 37:498-504.

10. Camargo R, Pacagnella R, Cecatti J, Parpinelli M, Souza J, Sousa M. Subsequent reproductive outcome in women who have experienced a potentially life-threatening condition or a maternal near-miss during pregnancy. Clinics (São Paulo) 2011; 66:1367-72.

11. Acelas-Granados DF, Orostegui A, AlarcónNivia MA. Factores de riesgo para morbilidad materna extrema en gestantes sin demora en la atención médica según la estrategia camino para la supervivencia. Rev Chil Obstet Ginecol 2016; 81:181-8.

12. Thaddeus S, Maine D. Too far to walk: maternal mortality in context. Soc Sci Med 1994; 38:1091-110. 
13. Szulik D, Szwarc L. Era una bomba de tiempo: el derrotero de la morbilidad materna severa en el Área Metropolitana de Buenos Aires. Salud Colect 2015; 11:553-64.

14. Rodríguez E, Aguilar P, Montero L, Hoil J, Andueza G. Demoras en la atención de complicaciones maternas asociadas a fallecimientos en municipios del sur de Yucatán, México. Rev Bioméd (México) 2012; 23:23-32.

15. World Health Organization. Evaluating the quality of care for severe pregnancy complications. The WHO near-miss approach for maternal health. Geneva: World Health Organization; 2011.

16. Strauss A, Corbin J. Bases de la investigación cualitativa, técnicas y procedimientos para desarrollar la teoría fundamentada. Bogotá: Editorial Universidad de Antioquia; 2002.

17. Yepes C, Molina D. La comparación en el análisis de la investigación cualitativa con teoría fundada. Rev Fac Nac Salud Pública 2015; 33 Suppl 1:S90-2.

18. Domingues RMSM, Dias MAB, Schilithz AOC, Leal MC. Factors associated with maternal near miss in childbirth and the postpartum period: findings from the birth in Brazil National Survey, 2011-2012. Reprod Health 2016; 13 Suppl 3:115

19. Dias MAB, Domingues RMSM, Schilithz AOC, Nakamura-Pereira M, Diniz CSG, Brum IR, et al. Incidência do near miss materno no parto e pós-parto hospitalar: dados da pesquisa Nascer no Brasil. Cad Saúde Pública 2014; 30 Suppl 1:S169-81.

20. González RD, Vélez GA, Agudelo SM, Gómez J, Wylie J. Características de la atención hospitalaria y su relación con la morbilidad materna extrema en Medellín, Colombia. Rev Panam Salud Pública 2014; 35:15-22.

21. Abalos E, Cuesta C, Carroli G, Qureshi Z, Widmer M, Vogel JP, et al. Pre-eclampsia, eclampsia and adverse maternal and perinatal outcomes: a secondary analysis of the World Health Organization Multicountry Survey on Maternal and Newborn Health. BJOG 2014; 121 Suppl 1:14-24.

22. Hernández J, Avila L, Valencia A, Poblano O. Evaluación inicial del Seguro Popular sobre gasto catastrófico en salud en México. Rev Salud Pública 2008; 10:18-32.
23. Villaseñor R, García M, Ramírez H, León S Aptitud clínica del médico familiar sobre preeclampsia-eclampsia en la unidad médica familiar no. 94 del IMSS. Atención Familiar 2017; 24:27-31.

24. Aguiar C, Tanaka AC. Memórias coletivas de mulheres que vivenciaram o near miss materno: necessidades de saúde e direitos humanos. Cad Saúde Pública 2016; 32:e0161215.

25. Mackintosh N, Rance S, Carter W, Sandall J. Working for patient safety: a qualitative study of women's help-seeking during acute perinatal events. BMC Pregnancy Childbirth 2017; 17:232.

26. Norhayati MN, Hazlina NHN, Asrenee AR, Sulaiman Z. The experiences of women with maternal near miss and their perception of quality of care in Kelantan, Malaysia: a qualitative study. BMC Pregnancy Childbirth 2017; 17:189.

27. Laza C, Quintero J, Jiménez HJ, Preciado JA. Experiencias y sentimientos vividos durante la gestación de alto riesgo: un estudio documental. Enferm Glob 2013; 12357-71.

28. Bittencourt SDA, Reis LGC, Ramos MM, Rattner D, Rodrigues PL, Neves DCO, et al. Estrutura das maternidades: aspectos relevantes para a qualidade da atenção ao parto e nascimento. Cad Saúde Pública 2014; 30 Suppl 1:S208-19.

29. Molina AN. Territorio, lugares y salud: redimensionar lo espacial en salud pública. Cad Saúde Pública 2018; 34:e00075117.

30. Rodríguez E, Palma M, Zapata R. Causas de demora en la atención de pacientes con complicaciones obstétricas ¿qué es necesario atender? Ginecol Obstet Méx 2014; 82:647-58.

31. Cecatti J, Souza R, Pacagnella R, Leal M, Moura E, Santos L. Morbilidad materna extremadamente grave entre las usuarias del sistema de salud pública en las regiones amazónica y noreste del Brasil. Rev Panam Salud Pública 2015; 37:232-8.

32. Karolinski A, Mercer R, Micone P, Ocampo C, Mazzoni A, Fontana O, et al. The epidemiology of life-threatening complications associated with reproductive process in public hospitals in Argentina. Br J Obstet Gynecol 2013; 120:1685-95 


\section{Abstract}

The objectives were to analyze the experiences of survivors of maternal near miss based on the three delays model and to identify the obstacles to this process in the state of San Luis Potosí, Mexico. In 2016-2017, a qualitative study was performed with 27 women who had experienced maternal near miss in public hospitals between 2014 and 2016. The informants were selected with theoretical sampling and interviewed at their homes. Data analysis was based on the theoretical framework proposed by Strauss or Corbin. The categories associated with the first delay were: (1) missed opportunities for identification of maternal near miss; (2) failure of the women to voice their symptoms out of fear of reprimand; (3) dilemma between fear and the faith that a higher power will solve the problem; and (4) development of the perception of obstetric risk. The second delay involved: (1) lack of urban transportation infrastructure to access healthcare services; (2) lack of regulation of transportation costs; (3) lack of clarity on itineraries and critical routes to access care; and (4) lack institutional coordination for timely transportation. The third delay involved: (1) institutional refusal of care; (2) deficient infrastructure, personnel, and equipment; and (3) lack of expertise and experience in the healthcare personnel. The shared experiences reveal challenges for public policies and inter-sector work to improve the results in maternal near miss.

Healthcare Near Miss; Pre-Eclampsia; Maternal Health; Emergencies; Qualitative Research

\section{Resumo}

O objetivo foi analisar as experiências de sobreviventes de morbidade materna extrema para, a partir da abordagem das três demoras, identificar os determinantes que obstaculizam esse processo no estado de San Luis Potosí, México. No período 2016-2017 foi efetuado um estudo qualitativo com 27 mulheres que experimentaram um evento deste tipo, entre 2014 e 2016, em hospitais públicos. As informantes foram selecionadas por meio de uma amostragem teórica e foram entrevistadas em seus domicílios. A análise de dados foi feita através da proposta da teoria fundamentada de Strauss ef Corbin, as categorias associadas com a primeira demora foram: (1) oportunidades perdidas para a identificação de morbidade materna extrema, (2) temor em comunicar o mal estar frente à desqualificação, (3) entre o medo e a fé aguardando em que algo superior achará a solução, (4) desenvolvimento da percepção do risco obstétrico. Com a segunda demora: (1) falta de infraestrutura urbana para a transferência, (2) ausência de regulação de custos de transferência, (3) falta de transparência acerca dos itinerários e rumos críticos para acessar ao atendimento, (4) coordenação institucional para a transferência oportuna; e finalmente com a terceira: (1) rejeição institucional, (2) deficiências da infraestrutura, equipe e equipamento, e (3) entre a esperteza e a falta de experiência da equipe de saúde. As experiências compartilhadas evidenciam desafios ao respeito de política pública e trabalho intersetorial para melhorar os resultados em matéria de morbidade materna extrema.

Near Miss; Pré-Eclâmpsia; Saúde Materna;

Emergências; Pesquisa Qualitativa

Recibido el 25/Feb/2018

Versión final presentada el 27/Feb/2019

Aprobado el 04/Abr/2019 